\title{
Brownian motion, hydrodynamics, and the osmotic pressure
}

\author{
John F. Brady \\ Department of Chemical Engineering, California Institute of Technology, Pasadena, California 91125
}

(Received 25 August 1992; accepted 10 November 1992)

\begin{abstract}
It is shown that the osmotic pressure of a colloidal dispersion can be interpreted as the isotropic part of the macroscopic particle stress in the suspension. The particle stress is in turn expressible in terms of hydrodynamic interactions among the suspended particles. Thus, there is a completely mechanical definition of the osmotic pressure, just as there is for the pressure in a molecular fluid. For an equilibrium suspension of colloidal particles subjected to thermal Brownian forces, this mechanical definition is shown to be exactly equal to the usual "thermodynamic" one. The derivation given here places the equilibrium and nonequilibrium properties of macroparticle fluids on the same mechanical foundation that underlies the statistical mechanics of simple liquids. Furthermore, through this development the relationship between hydrodynamics and kinetic-theory-like descriptions of colloids is explained.
\end{abstract}

\section{INTRODUCTION}

Since the work of Einstein on Brownian motion it is well known that colloidal particles dispersed in a fluid of smaller molecules behave as a thermodynamic system. The structure and properties of this "macroparticle system" are completely determined by the forces of interaction among the macroparticles; the solvent or fluid only enters in the form of a thermal bath providing $k T$ of energy for each degree of freedom of the macroparticles-the well-known fluctuation-dissipation theorem. ${ }^{1-3}$ This model underlies virtually all treatments of colloids, polymers, microemulsions, micells, etc., in short almost all "complex fluids." The correctness and accuracy of this description has been well verified experimentally, particularly in the recent experiments of Vrij and co-workers, ${ }^{4,5}$ who have measured the structure factor for spherical colloidal particles ranging in size from 20 to $200 \mathrm{~nm}$. Indeed, the idealized hardsphere fluid can be realized more accurately and probed more easily with colloids than with its atomic realization argon.

The equation of state for a colloidal system is expressed in terms of the osmotic pressure $\Pi$. The osmotic pressure is defined as the negative derivative of the Helmholtz free energy with respect to volume at constant temperature. Making use of standard statistical mechanical relations between free energy, partition function, and interparticle potential $V$, the osmotic pressure becomes ${ }^{3,6}$

$$
\Pi=n k T-\frac{2 \pi}{3} n^{2} \int_{0}^{\infty} r^{3} g(r) \frac{d V}{d r} d r
$$

where $n$ is the number density of colloidal particles, and $g(r)$ is the pair-distribution function. We have also assumed that the interparticle potential is pairwise additive, although this restriction is not essential. It is important to note that the potential here is the potential between the macroparticles; the solvent does not enter. In the idealized case of hard spheres, the interparticle potential is zero except if particles were to overlap where the potential is in- finite. This gives a $\delta$ function at contact and the osmotic pressure becomes

$$
\frac{\Pi}{n k T}=1+4 \phi g(2),
$$

where $\phi=4 \pi n a^{3} / 3$ is the volume fraction of particles of radii $a$. Only the contact value of the pair-distribution function, $g(r=2)$, determines the pressure.

The power and beauty of this thermodynamic development is that the equilibrium properties of colloidal dispersions, or any macroparticle system, can be determined without reference to the solvent. Indeed, Eqs. (1) and (2) are identical to those for the pressure of a molecular liquid. However, in a molecular fluid there is also a purely mechanical definition of pressure that follows directly from the equations of motion and makes no appeal to any thermodynamic arguments. Correspondingly for colloids, there should be an analogous definition of the osmotic pressure derivable from the mechanical equations of motion of the macroparticles. It is the purpose of this paper to provide such a mechanical definition and derivation. We shall see that the equations of motion of the macroparticles involve hydrodynamic interactions and thus the mechanical definition of the osmotic pressure is hydrodynamical in origin. We shall show explicitly, however, that the same "thermodynamic" formula, Eq. (1), results.

This derivation of the osmotic pressure from hydrodynamics also connects properly to studies of nonequilibrium, or transport, properties of colloids. Traditionally, equilibrium properties of colloidal dispersions are explained and derived by appealing to the thermodynamic arguments that led to Eq. (1). When transport properties, such a diffusion, rheology, etc., are studied, however, one needs to bring in a description of the dynamics of particle motion and thus hydrodynamics appear. One is left then with an uncomfortable merger of two descriptions-a thermodynamic one for the equilibrium free energy where the solvent does not appear, and a hydrodynamic one for the particle motion where the solvent is treated as a continuum fluid. These two descriptions are then simply added to- 
gether. All studies of complex fluids proceed on this basis. In the derivation presented here, the mechanical equations of motion are taken as fundamental and both the thermodynamic and transport properties follow by statistical averaging, just as in a molecular fluid. Furthermore, through this derivation we shall be able to explain why some recent kinetic theory studies of colloids, in which the hydrodynamics are ignored, may give reasonable, qualitative, results. ${ }^{7,8}$

In the next section we present the mechanical equations of motion for the macroparticles, which are the wellknown Langevin equations, and the definition of the macroscopic stress. This will first be done for hard spheres, where it will be seen that the evolution equations and the macroscopic properties are expressed entirely in terms of hydrodynamic interactions. From these we shall derive Eq. (2) for the osmotic pressure. In the following section we generalize the results to include any type of interparticle force and obtain Eq. (1) for the osmotic pressure. Finally, in Sec. III we shall generalize to nonequilibrium situations and show the correspondence between kinetic theory treatments of colloidal dispersions and hydrodynamics.

\section{THE MECHANICAL EQUATIONS OF MOTION}

\section{A. Hard spheres}

The macroparticles are modeled as rigid spheres immersed in an incompressible continuum solvent of viscosity $\eta$ and density $\rho$. The equations of motion for the particles are the coupled $N$-body Langevin equation: ${ }^{9,10}$

$$
\mathbf{m} \cdot \frac{d \mathbf{U}}{d t}=\mathbf{F}^{H}+\mathbf{F}^{B},
$$

which simply states: mass $\times$ acceleration equals the sum of the forces. In Eq. (3) $\mathrm{m}$ is a generalized mass/moment of inertia matrix of dimension $6 N \times 6 N$, $\mathrm{U}$ is the particle translational/rotational velocity vector of dimension $6 N$, and the $6 N$ force/torque vectors $\mathbf{F}$ represent: (1) the hydrodynamic forces $\mathrm{F}^{H}$ exerted on the particles due to their motion relative to the fluid, and (2) the stochastic forces $\mathbf{F}^{B}$ that give rise to Brownian motion.

The particle motion occurs at low Reynolds number $(\rho U a / \eta \ll 1$ ) so that the hydrodynamic drag force will be linear in the velocities:

$$
\mathbf{F}^{H}=-\mathbf{R}_{F U} \cdot \mathbf{U}
$$

where $\mathbf{R}_{F U}$ is the hydrodynamic resistance tensor that characterizes the hydrodynamic interactions among the particles. $\mathbf{R}_{F U}(\mathbf{x})$ is a purely geometric quantity that depends on the configuration $\mathbf{x}$ of the particles only., ${ }^{9,11,12}$ For a single isolated sphere $\mathbf{R}_{F U}=6 \pi \eta a \mathbf{I}$, with $\mathbf{I}$ the isotropic tensor, which is the well-known Stokes drag law.

The stochastic or Brownian force $\mathbf{F}^{B}$ arises from the thermal fluctuations in the solvent and is characterized by

$$
\left\langle\mathbf{F}^{B}\right\rangle=0 \quad \text { and }\left\langle\mathbf{F}^{B}(0) \mathbf{F}^{B}(t)\right\rangle=2 k T \mathbf{R}_{F U} \delta(t) .
$$

In Eq. (5) the angle brackets denote an ensemble average, $k$ is Boltzmann's constant, $T$ is the absolute temperature, and $\delta(t)$ is the delta function. The amplitude of the corre- lation between the Brownian forces at time 0 and at time $t$ results from the fluctuation-dissipation theorem for the $N$-body system.

Equations (3)-(5) provide a complete description of the motion of the macroparticles. The key assumptions that have been made are that the solvent can be modeled as a continuum fluid, that the hydrodynamic forces are given by their low-Reynolds-number values, and that the stochastic Brownian forces have zero mean and are correlated instantaneously in time. The motion of the macroparticles occurs on a time scale $\tau \sim m / 6 \pi \eta a$, which is long compared to that of the solvent provided the particle size is large compared to that of a solvent molecule. The amplitude of the correlation in the Brownian forces is chosen so that the mean kinetic energy imparted to the macroparticles is $k T$, i.e.,

$$
\frac{1}{2}\langle\mathbf{U} \cdot \mathbf{m} \cdot \mathbf{U}\rangle=\frac{1}{2} k T \mathbf{I},
$$

which can be shown by straightforward integration of Eq. (3).

The only connection made to the thermal bath provided by the solvent, and therefore to the thermal temperature $k T$, is through the correlation of the Brownian forces. We could equally well place any constant in front of $\mathbf{R}_{F U}$ in Eq. (5) and the resulting dynamics would be unchanged. The macroparticles are only aware of the true thermal temperaturc $k T$ through this scale factor. This means that we can take Eqs. (3)-(5) to be the primitive, fundamental, equations of motion and thus define the macroparticle mechanical system as that system which evolves according to these equations. The thermodynamics or statistical mechanics of this "new" macroparticle system will now follow from these equations and be completely specified by them. No reference to free energy, etc., need be made; indeed, they are derivable from Eqs. (3)-(5).

One may object that this argument is somewhat circular in that fluctuating Brownian forces characterized by Eq. (5) will reproduce conventional thermodynamics and thus lead naturally to Eq. (2) for the osmotic pressure. While this is indeed true and served as the original motivation for the Langevin equation with Eq. (5) for the random forces, if we place ourselves in the position of someone who does not know this connection, but rather only knows the mechanical evolution equations (3)-(5), then our task is to deduce the statistical properties from the equations of motion. From this perspective it is not at all obvious that Eqs. (3)-(5), and the macroscopic stress (7) below, will lead to Eq. (2) for the osmotic pressure. In fact, even if we know conventional statistical mechanics and therefore wish to use this apparatus to infer the macroscopic properties of a dynamical system, the forces in Eq. (3) are not derivable from a potential, there is no Hamiltonian, and conventional statistical mechanics is, therefore, of little use. Nevertheless, a well-defined statistical mechanics exists and Eq. (2) does follow from Eqs. (3)-(5) as we now show. It is in this sense that our analysis provides a new mechanical perspective on macroparticle dispersions and a derivation of their thermodynamics. 
To determine the average or macroscopic properties of a colloidal dispersion, and in particular the osmotic pressure, we need an expression for the particles' contribution to the macroscopic stress $\langle\Sigma\rangle$. The macroscopic particle stress in a hard-sphere dispersion evolving under the action of Brownian forces only is given by ${ }^{9,10,13}$

$$
\langle\Sigma\rangle=-n\langle\mathbf{U} \cdot \mathbf{m} \cdot \mathbf{U}\rangle+n\left\langle\mathbf{S}^{B}\right\rangle .
$$

The first term on the right-hand-side of Eq. (7) is the usual "kinetic" contribution to the stress, called a Reynolds stress in continuum mechanics. From Eq. (6) this term is simply $-n k T$ I.

The second term $\mathbf{S}^{B}$ is the particle stresslet due to the fluctuating Brownian forces. The stresslet is the symmetric first moment of the hydrodynamic force distribution integrated over the particle surface, and is a measure of the resistance of the particles to deformation. [The antisymmetric first moment is the torque and has already been included in Eq. (3). We have assumed that there is no net external torque acting on the particles so that the particle stress is symmetric. If external torques do act, there is an antisymmetric contribution to the stress proportional to the average torque (cf. Sec. III).] We can determine the Brownian stresslet by writing a Langevin equation for the symmetric first moment as we did for the forces/torques in Eq. (3). Since the particles are rigid and do not deform, the Langevin equation for the stresslets becomes

$$
0=\mathbf{S}^{H}+\mathbf{S}^{B} \text {, }
$$

which simply says that the fluctuating Brownian stresslets are equal and opposite to the hydrodynamic stresslets, $\mathbf{S}^{H}$. Again, from low-Reynolds number hydrodynamics the hydrodynamic stresslet is linearly related to the particle velocities through another hydrodynamic resistance function:

$$
\mathbf{S}^{H}=-\mathbf{R}_{S U} \cdot \mathbf{U} \text {, }
$$

where the subscript notation should now be obvious. Using Eqs. (8) and (9) with the Langevin equation (3) for the velocities, integrating over the fast time scale of the fluctuating Brownian forces, and taking into account the variation of $\mathbf{R}_{S U}$ and $\mathbf{R}_{F U}$ with configuration, gives for the Brownian stresslet: ${ }^{10}$

$$
\mathbf{S}^{B}=-k T \nabla \cdot\left(\mathbf{R}_{S U} \cdot \mathbf{R}_{F U}^{-1}\right),
$$

where the configuration-space divergence is with respect to the last index of $\mathbf{R}_{F U}^{-1}$. Thus, the macroscopic stress becomes

$$
\langle\Sigma\rangle=-n k T \mathbf{I}-n k T\left\langle\nabla \cdot\left(\mathbf{R}_{S U} \cdot \mathbf{R}_{F U}^{-1}\right)\right\rangle .
$$

In the absence of any external forcing, such as a shearing motion for example, the macroscopic stress is isotropic and the mechanical definition of the osmotic pressure is simply the trace of $\Sigma$ :

$$
\mathbf{I}:\langle\Sigma\rangle=-3 \Pi \text {, }
$$

or

$$
\Pi=n k T+n k T\langle\nabla \cdot \mathbf{B}\rangle,
$$

where

$$
\mathbf{B}=\frac{1}{3} \mathbf{I}: \mathbf{R}_{S U} \cdot \mathbf{R}_{F U}^{-1}
$$

We have now arrived at a description of the macroparticle system where the equations of motion and the osmotic pressure (and any other dynamical property) are completely defined (with the scale factor $k T$ ) in terms of $h y$ drodynamic interactions. Well defined methods exist for computing these hydrodynamic resistance functions that are accurate from infinite dilution to close packing, ${ }^{9-11}$ but for our purposes here, we shall not need to know very much about these functions. The reader should note that in previous work the stresslets were defined to be symmetric and traceless, but in the convention adopted here the trace of $\mathbf{S}^{H}$ is not zero; i.e.,

$$
\mathbf{P} \equiv \mathbf{I}: \mathbf{R}_{S U}
$$

is not zero. $\mathbf{P}$, the hydrodynamic resistance function relating the particle pressure to the velocities, has recently been determined. ${ }^{14}$

The determination of the osmotic pressure involves the computation of the equilibrium average of $\boldsymbol{\nabla} \cdot \mathbf{B}$. This can be carried out explicitly, numerically, through the hydrodynamic functions, but considerably greater insight can be gained by performing the average analytically. To do this we make use of the evolution equation for the $N$-particle distribution function, $P_{N}$. Corresponding to the $N$-body Langevin equation (3) is the $N$-body Smoluchowski equation for the configuration-space distribution function: $:^{3,9}$

$$
\frac{\partial P_{N}}{\partial t}+\nabla \cdot \mathrm{j}_{N}=0
$$

with the probability flux $\mathbf{j}_{N}$ given by

$$
\mathbf{j}_{N}=-\mathbf{D} \cdot \nabla P_{N} \text {. }
$$

The $N$-particle diffusivity $\mathbf{D}$ is related to the hydrodynamic resistance function through the Stokes-Einstein relation

$$
\mathbf{D}=k T \mathbf{R}_{F U}^{-1} \text {. }
$$

On time scales long compared to the Brownian relaxation time, $\tau=m / 6 \pi \eta a$, the motion of the particles described by the Langevin equation ( 3 ) is entirely equivalent to that described by the evolution of the probability density from the Smoluchowski equation.

At cquilibrium the flux $\mathbf{j}_{N} \equiv 0$ and therefore

$\nabla P_{N}=0$ at all points in the volume.

Returning to Eq. (12) for the osmotic pressure, we can write in the limit of large $N$

$$
\begin{aligned}
\langle\nabla \cdot \mathbf{B}\rangle & =\frac{1}{N} \sum_{\alpha=1}^{N} \nabla_{\alpha} \cdot \mathbf{B}_{\alpha} \\
& =\frac{1}{N} \frac{1}{N !} \sum_{\alpha=1}^{N} \int\left(\nabla_{\alpha} \cdot \mathbf{B}_{\alpha}\right) P_{N} d \mathbf{r}_{N} .
\end{aligned}
$$

Now,

$$
\left(\nabla_{\alpha} \cdot \mathbf{B}_{\alpha}\right) P_{N}=\nabla_{\alpha} \cdot\left(\mathbf{B}_{\alpha} P_{N}\right)-\mathbf{B}_{\alpha} \cdot \nabla_{\alpha} P_{N},
$$

and with Eq. (17) and using the divergence theorem, Eq. (18) becomes 


$$
\begin{aligned}
\langle\boldsymbol{\nabla} \cdot \mathbf{B}\rangle & =\frac{1}{N} \frac{1}{N !} \sum_{\alpha=1}^{N} \oint \mathbf{n}_{\alpha} \cdot \mathbf{B}_{\alpha} P_{N} d S_{N} \\
& =\oint \mathbf{n}_{2} \cdot\left\langle\mathbf{B}_{2}\right\rangle_{2} P_{2}\left(\mathbf{r}_{2} \mid \mathbf{r}_{1}\right) d S_{2},
\end{aligned}
$$

where $\left\langle\mathbf{B}_{2}\right\rangle_{2}$ is the conditionally averaged value of $\mathbf{B}$ with two particles fixed, $\mathbf{n}_{2}$ is the normal along the line connecting the centers of particles 1 and 2 , and $S_{2}$ is the surface area of contact between particles 1 and 2 . Noting that $P_{2}\left(\mathrm{r}_{2} \mid \mathbf{r}_{1}\right)=n g(\mathbf{r}), \mathbf{r}=\mathbf{r}_{2}-\mathbf{r}_{1}, d S_{2}=(2 a)^{2} 4 \pi$, and the fact that the equilibrium structure is isotropic, Eq. (19) becomes

$$
\frac{\Pi}{n k T}=1-4 \phi g(2) \overline{\left\langle\mathbf{n}_{2} \cdot \mathbf{B}_{2}\right\rangle_{2}},
$$

where

$$
\overline{\left\langle\mathbf{n}_{2} \cdot \mathbf{B}_{2}\right\rangle_{2}}=\frac{3}{a}\left\langle\mathbf{n}_{2} \cdot \mathbf{B}_{2}(r=2)\right\rangle_{2} .
$$

Comparing Eqs. (21) to (2), we see that the two expressions for the osmotic pressure for hard spheres will agree if $\overline{\left\langle\mathbf{n}_{2} \cdot \mathbf{B}_{2}\right\rangle_{2}}=1$. This we shall now show to be the case.

From low-Reynolds-number hydrodynamics, or more generally from lubrication theory, ${ }^{14,15}$ the hydrodynamic stresslet at contact, $-\mathbf{R}_{S U} \cdot \mathbf{U}$, as the separation between the particle surfaces approaches zero, is directly proportional to the hydrodynamic force, $\mathbf{F}^{H}=-\mathbf{R}_{F U} \cdot \mathbf{U}$. This comes from the singular form of the resistance tensors at contact. Both $\mathbf{R}_{F U}$ and $\mathbf{R}_{S U}$ are singular as $1 / \xi$ as $\xi \rightarrow 0$, where $\xi=r-2$, corresponding to the large localized pressure required to pump out the viscous fiuid separating the particles as their surfaces approach one another. Since $\mathbf{R}_{S U}$ gives the first moment of the force distribution, and all the singular force is localized at the point of contact,

$$
\mathbf{R}_{S U} \rightarrow a \mathbf{d R} \mathbf{R}_{F U} \text { as }\left|\mathbf{r}_{2}-\mathbf{r}_{1}\right| \rightarrow 2,
$$

where $d=\left(\mathbf{r}_{2}-\mathbf{r}_{1}\right) /\left|\mathbf{r}_{2}-\mathbf{r}_{1}\right|$ is the unit vector along the line of centers of any two particles. Thus, at contact $r=2$,

$$
\mathbf{P}=\mathbf{I}: \mathbf{R}_{S U}=a \mathbf{d} \cdot \mathbf{R}_{F U}
$$

and

$$
\mathbf{B}=\frac{1}{3} \mathbf{P} \cdot \mathbf{R}_{F U}^{-1}=\frac{a}{3} \mathbf{d} .
$$

Hence,

$$
\overline{\left\langle\mathbf{n}_{2} \cdot \mathbf{B}_{2}\right\rangle_{2}}=\frac{3}{a}\left\langle\mathbf{n}_{2} \cdot \mathbf{B}_{2}(r=2)\right\rangle_{2} \equiv 1,
$$

and Eq. (20) is identical to Eq. (2).

We have thus derived the osmotic pressure for colloidal hard spheres from a purely mechanical perspective in which all quantities were defined in terms of hydrodynamics. No appeal to thermodynamics has been made, apart from accepting $k T$ as the amplitude for the fluctuating Brownian forces. Furthermore, although we have considered hard spheres, there was no need to introduce the hardsphere interparticle potential. The hydrodynamics alone are sufficient to determine the particle dynamics and to guarantee that particles never overlap. This is so because of the strong lubrication forces referred to above. Indeed, if we had included the hard-sphere interparticle potential this would give rise to a nonzero force on the particles only when two particles touch. However, since the relative velocity of the particles resulting from this force would be zero because their relative mobility is zero on contact [i.e., $\mathbf{R}_{F U}^{-1} \cdot \nabla V=0$ for $\left.\nabla V \sim-\mathbf{n} \delta(r-2)\right]$; a hard-sphere potential plays no dynamical role with hydrodynamic interactions. Evidently, the no-slip hydrodynamic boundary condition on the surfaces of the particles ensures that they behave as hard spheres. Also, it should be noted that the fact that the particles were presumed spherical and monodisperse is not important; $\langle\boldsymbol{\nabla} \cdot \mathbf{B}\rangle$ is still given by Eq. (19) and the geometric generalization of Eq. (22) still holds.

\section{B. Interparticle forces}

The generalization of the preceding derivation to include interparticle forces (or external ones for that matter) is very straightforward. We shall assume that the forces are derivable from a potential $V_{p}$. This potential need not be pairwise additive. It is, however, a potential that gives rise to colloidal-scale forces, for example, charged electrostatic, etc. Thus, on each colloidal particle a force

$$
\mathbf{F}^{P}=-\nabla V_{P}(\mathbf{x})
$$

acts, where on particle 1 the gradient would be with respect to the coordinates of particle 1. Interparticle torques could also be included in Eq. (24). With these forces the Langevin equation (3) becomes simply

$$
\mathbf{m} \cdot \frac{d \mathbf{U}}{d t}=\mathbf{F}^{H}+\mathbf{F}^{B}+\mathbf{F}^{P}
$$

and the particle stress (7) now reads as

$$
\langle\Sigma\rangle=-n\langle\mathbf{U} \cdot \mathbf{m} \cdot \mathbf{U}\rangle+n\left\langle\mathbf{S}^{B}\right\rangle+n\left\langle\mathbf{S}^{P}\right\rangle
$$

with

$$
\left\langle\mathbf{S}^{P}\right\rangle=-\left\langle\left(\mathbf{x I}+\mathbf{R}_{S U} \cdot \mathbf{R}_{F U}^{-1}\right) \cdot \mathbf{F}^{P}\right\rangle .
$$

The contribution to the macroscopic stress from the interparticle forces has the familiar $\left\langle\mathbf{x F}^{P}\right\rangle$ term, just as in a molecular liquid. The other term proportional to $\mathbf{R}_{S U}$ arises from the hydrodynamic stresses generated in the fluid caused by the motion of the particles under the action of the interparticle forces. ${ }^{9,10,13}$ The motions are superimposable because we are at low Reynolds numbers. Also, the kinetic stress contribution $\langle\mathbf{U} \cdot \mathbf{m} \cdot \mathbf{U}\rangle$ always remains $k T \mathbf{I}$; variations from this value are due to particle inertia which are neglected at low Reynolds numbers.

The osmotic pressure now reads

$$
\Pi=n k T+n k T\langle\nabla \cdot \mathbf{B}\rangle+n k T\left\langle(\mathbf{x}+\mathbf{B}) \cdot \frac{\mathbf{F}^{P}}{k T}\right\rangle \text {. }
$$

To obtain Eq. (1) from (28) we proceed as before with the Smoluchowski equation (15), where all that changes is the constitutive law for the flux $\mathbf{j}_{N}$ :

$$
\mathbf{j}_{N}=\mathbf{D} \cdot\left[\frac{\mathbf{F}^{P}}{k T}-\nabla \ln P_{N}\right] P_{N}
$$


The definition of equilibrium is that the flux $\mathbf{j}_{N} \equiv 0$, which means that the quantity in square brackets must be zero as $D$ is positive definite. At equilibrium we recover the familiar Boltzmann distribution

$$
P_{N}=P_{N}^{n} \exp \left(-V_{P} / k T\right) \text {. }
$$

As before we have for $\langle\boldsymbol{\nabla} \cdot \mathbf{B}\rangle$ :

$$
\langle\nabla \cdot \mathbf{B}\rangle=\frac{1}{N} \frac{1}{N !} \sum_{\alpha=1}^{N} \int\left[\nabla_{\alpha} \cdot\left(\mathbf{B}_{\alpha} P_{N}\right)-\mathbf{B}_{\alpha} \cdot \nabla_{\alpha} P_{N}\right] d \mathbf{r}_{N}
$$

Although $\nabla P_{N} \neq 0$ as for hard spheres, we can write the interparticle force contribution to the stress as a probability integral also,

$$
\begin{aligned}
& \left\langle(\mathbf{x}+\mathbf{B}) \cdot \frac{\mathbf{F}^{P}}{k T}\right\rangle \\
& =\frac{1}{N} \frac{1}{N !} \sum_{\alpha=1}^{N} \int\left[\mathbf{x}_{\alpha} \cdot \frac{\mathbf{F}_{\alpha}^{P}}{k T}+\mathbf{B}_{\alpha} \cdot \frac{\mathbf{F}_{\alpha}^{P}}{k T}\right] P_{N} d \mathbf{r}_{N} .
\end{aligned}
$$

Combining Eqs. (31) and (32), we have

$$
\begin{aligned}
\langle\nabla \cdot \mathbf{B}\rangle & +\left\langle(\mathbf{x}+\mathbf{B}) \cdot \frac{\mathbf{F}^{P}}{k T}\right\rangle \\
= & \frac{1}{N} \frac{1}{N !} \sum_{\alpha=1}^{N} \int\left[\nabla_{\alpha} \cdot\left(\mathbf{B}_{\alpha} P_{N}\right)+\mathbf{x}_{\alpha} \cdot \frac{\mathbf{F}_{\alpha}^{P}}{k T} P_{N}\right] d \mathbf{r}_{N} \\
& +\frac{1}{N} \frac{1}{N !} \sum_{\alpha=1}^{N} \int \mathbf{B}_{\alpha} \cdot\left[\frac{\mathbf{F}_{\alpha}^{P}}{k T}-\nabla_{\alpha} \ln P_{N}\right] P_{N} d \mathbf{r}_{N} .
\end{aligned}
$$

The second integral on the right-hand-side of Eq. (33) is identically zero at equilibrium because the quantity in square brackets is zero. The $\mathbf{B}$ part of the first integral on the right-hand side is just what we had for hard spheres, and thus Eq. (33) gives for the osmotic pressure

$$
\begin{aligned}
\frac{\Pi}{n k T} & =1+4 \phi g(2)+\int\left\langle\mathbf{x}_{2} \cdot \frac{\mathbf{F}_{2}^{P}}{k T}\right\rangle_{2} P_{2}\left(\mathbf{r}_{2} \mid \mathbf{r}_{1}\right) d \mathbf{r}_{2} \\
& =1+4 \phi g(2)-\int\left\langle\mathbf{x}_{2} \cdot \nabla_{2}\left(V_{P} / k T\right)\right\rangle_{2} P_{2}\left(\mathbf{r}_{2} \mid \mathbf{r}_{1}\right) d \mathbf{r}_{2}
\end{aligned}
$$

Equation (35) is identical to Eq. (1) when we note that in the thermodynamic argument that led to Eq. (1) the interparticle potential there, $V$, must also include the hardsphere part because no hydrodynamics were present. That is,

$$
V=V_{\mathrm{HS}}+V_{P}
$$

with $\nabla V_{\mathrm{HS}}=-\mathbf{n} \delta(r-2 a)$. [The presence of the hardsphere osmotic pressure is of no consequence when strong repulsive intcrparticlc forces are present because $g(r=2)$ will be zero, and all the osmotic pressure comes from the interparticle potential in Eq. (35).]

\section{GENERALIZATION TO NONEQUILIBRIUM FORCING}

We shall now generalize the above analysis to nonequilibrium situations, particularly the presence of an external flow. If the external forcing is in the form of an external body force, for example, gravity where the particles would settle, then the analysis and equations in the preceding section remain unchanged. New features only arise due to the presence of an external flow. The Langevin equation (3) is the same as with interparticle forces:

$$
\mathbf{m} \cdot \frac{d \mathbf{U}}{d t}=\mathbf{F}^{H}+\mathbf{F}^{B}+\mathbf{F}^{P},
$$

except that the hydrodynamic forces now include a contribution from the linear shearing flow:

$$
\mathbf{F}^{H}=-\mathbf{R}_{F U} \cdot(\mathbf{U}-\langle\mathbf{U}\rangle)+\mathbf{R}_{F E}:\langle\mathbf{E}\rangle .
$$

In Eq. (37) the external shearing motion is characterized by the bulk rate of strain tensor $\langle\mathbf{E}\rangle$ and the imposed flow $\langle\mathbf{U}\rangle=\langle\mathbf{G}\rangle \cdot \mathbf{x}$, where $\langle\mathbf{G}\rangle=\langle\nabla \mathbf{U}\rangle$, and $\langle\mathbf{E}\rangle=(1 / 2)[\langle\nabla \mathbf{U}\rangle$ $\left.+\langle\nabla \mathbf{U}\rangle^{\dagger}\right]$ is the symmetric part of $\langle\mathbf{G}\rangle$. The antisymmetric part corresponds to rotation and is included in the definition of the bulk angular velocity $\langle\Omega\rangle$. The new hydrodynamic resistance tensor $\mathbf{R}_{F E}$ gives the force/torque on the particles due to the shearing motion. The Brownian forces are unchanged from their equilibrium form (5). From the perspective of the solvent this is an assumption that the fluctuation-dissipation theorem remains unchanged when the fluid is out of equilibrium. From the perspective of the macroparticles, it is simply the definition of their mechanics.

Corresponding to this hydrodynamic shear force, the particle stress now becomes

$$
\langle\Sigma\rangle=-n k T \mathbf{I}+n\left[\left\langle\mathbf{S}^{H}\right\rangle+\left\langle\mathbf{S}^{B}\right\rangle+\left\langle\mathbf{S}^{P}\right\rangle\right],
$$

where $\mathbf{S}^{H}$ is the hydrodynamic stresslet due to the shearing motion. The stresslets are

$$
\begin{aligned}
& \left\langle\mathbf{S}^{H}\right\rangle=-\left\langle\mathbf{R}_{S U} \cdot \mathbf{R}_{F U}^{-1} \cdot \mathbf{R}_{F E}-\mathbf{R}_{S E}\right\rangle:\langle\mathbf{E}\rangle, \\
& \left\langle\mathbf{S}^{B}\right\rangle=-k T\left\langle\boldsymbol{\nabla} \cdot\left(\mathbf{R}_{S U} \cdot \mathbf{R}_{F U}^{-1}\right)\right\rangle, \\
& \left\langle\mathbf{S}^{P}\right\rangle=-\left\langle\left(\mathbf{x I}+\mathbf{R}_{S U} \cdot \mathbf{R}_{F U}^{-1}\right) \cdot \mathbf{F}^{P}\right\rangle .
\end{aligned}
$$

To get the complete macroscopic stress of the mixture of macroparticles and fluid, we would also need to add to Eq. (38) the contribution of the solvent or Auid, $\left\langle\Sigma_{f}\right\rangle$, which is just a constant hydrostatic pressure and the Newtonian viscosity:

$$
\left\langle\Sigma_{f}\right\rangle=-\left\langle p_{f}\right\rangle \mathbf{I}+2 \eta\langle\mathbf{E}\rangle .
$$

To complete the description, the Smoluchowski equation (15) is as before with the flux $\mathbf{j}_{N}$ :

$\mathbf{j}_{N}=\langle\mathbf{U}\rangle P_{N}+\mathbf{R}_{F U}^{-1} \cdot\left[\mathbf{R}_{F E^{*}}\langle\mathbf{E}\rangle+\mathbf{F}^{P}-k T \nabla \ln P_{N}\right] P_{N}$.

By straightforwardly working with $P_{N}$ as before, Eq. (38) for the particle stress may be written as 


$$
\begin{aligned}
\langle\Sigma\rangle= & -n k T \mathbf{I}-n k T a \int \mathbf{n}_{2} \mathbf{n}_{2} P_{2}\left(\mathbf{r}_{2} \mid \mathbf{r}_{1}\right) d S_{2}-n\left\langle\mathbf{x} \mathbf{F}^{P}\right\rangle \\
& -n\left\langle\mathbf{R}_{S U} \cdot \mathbf{R}_{F U}^{-1} \cdot \mathbf{R}_{F E}-\mathbf{R}_{S E}\right\rangle:\langle\mathbf{E}\rangle \\
& -n\left\langle\mathbf{R}_{S U} \cdot \mathbf{R}_{F U}^{-1} \cdot\left[\mathbf{F}^{P}-k T \nabla \ln P_{N}\right]\right\rangle
\end{aligned}
$$

Equation (42) is the exact form for the particle stress both in and out of equilibrium. In equilibrium, $\langle\mathbf{E}\rangle=0, \mathbf{F}^{P}$ $=k T \nabla \ln P_{N}$, and Eq. (42) reduces to the osmotic pressure derived above. Note that in Eq. (42) the angle brackets denoting the average must be carried out by weighting with the $N$-particle probability density $P_{N}$. This is so because $\nabla \ln P_{N}$ is only defined in the context of the Smoluchowski equation. This is in distinction to the averages implied in Eqs. (38)-(39c), where, at the Langevin level, all quantities are defined at each instant for all configurations. In Stokesian dynamics simulations, ${ }^{16}$ averages over particles and over time are used to compute the macroscopic properties; the probability density $P_{N}$ then follows by averaging, just as in a molecular fluid.

Equation (42) for the particle stress is very interesting in that there are five contributions to the bulk stress. The first term, $-n k T \mathrm{I}$, is the usual kinetic one and needs no further comment. In the second term, part of the direct Brownian contribution to the stress, $\mathbf{S}^{\bar{B}}$, has been transferred to knowledge of $g(\mathbf{r})$ at contact, as was the case in equilibrium. Note that no hydrodynamic functions appear here and that this integral does not involve any conditional averages. All that is needed is knowledge of how the contact value of $g(\mathbf{r})$ evolves as the shear rate changes. This term is identical to what one would get from a hard-sphere potential in a molecular liquid.

In the third term part of the interparticle force contribution, $\mathbf{S}^{P}$, has been cast into the familiar $\left\langle\mathbf{x F}^{P}\right\rangle$ stress of the same form as in molecular liquids. Note that $\left\langle\mathbf{x} \mathbf{F}^{P}\right\rangle$ need not be symmetric, and, in general, will not be if the interparticle forces are not central. In molecular fluids most interparticle forces are central, but for macroscale particles noncentral forces can easily arise as in the case of electrorheological fluids. ${ }^{17}$ Also, if there are external torques acting on the particles, the antisymmetric stress

$$
-n \epsilon \cdot\left\langle\mathbf{L}^{P}\right\rangle
$$

must be added to Eq. (38) or (42), where $\left\langle\mathbf{L}^{P}\right\rangle$ is the average external torque acting on the particles and $\epsilon$ is the unit alternating tensor. ${ }^{18}$

The fourth term is the hydrodynamic contribution to the stress, $\mathbf{S}^{H}$ in Eq. (39a). This term is purely hydrodynamic in origin and has no counterpart in a molecular fluid. It depends fundamentally on the finite size of the particles in that the resistance tensors $\mathbf{R}_{S U}$ and $\mathbf{R}_{S E}$ are proportional to $a^{3}$; for point particles these functions are zero. For a single isolated sphere, $\mathbf{R}_{S U}=0$ and $\left|\mathbf{R}_{S E}\right|$ $=(20 / 3) \pi \eta a^{3}$, which, when combined with Eq. (40) for the solvent, gives the well-known Einstein viscosity $\dot{\eta}_{\text {eff }}$ $=\eta[1+(5 / 2) \phi]$. The high-frequency dynamic viscosity of a colloidal dispersion is precisely this term with the average taken over the equilibrium structure. Stokesian dynamics calculations of this term are in excellent agreement with experiment for hard-sphere colloidal dispersions from infinite dilution up to volume fractions of $60 \% .^{19}$

While the high-frequency dynamic viscosity is one indication of the importance of the hydrodynamic contribution to the stress, at large shear rates the entire stress is dominated by hydrodynamics. That is, when the magnitude of the shearing forces become large compared to the Brownian forces (or interparticle forces), as characterized by the Péclet number $\mathrm{Pe}=6 \pi \eta \mathrm{a}^{3} \dot{\gamma} / k T$, both the microstructure and the stress are determined by hydrodynamics alone. Indeed, Eqs. (36)-(39a) are applicable for particles well in excess of $1 \mu \mathrm{m}$ in size where Brownian forces become insignificant. Stokesian dynamics simulations have accurately predicted the rheological (and other) response over a range in particle size from $10 \mathrm{~nm}$ to $1 \mathrm{~cm}$. This accuracy over 6 orders of magnitude in particle size demonstrates the accuracy of Stokesian dynamics, the robustness of the basic formulation, and the correctness of the underlying physics.

The final term in Eq. (42) gives the contribution to the particle stress due to the hydrodynamical stresses generated in the fluid resulting from the motion caused by the interparticle $\left(\mathbf{F}^{P}\right)$ and Brownian $\left(\nabla \ln P_{N}\right)$ forces. Again, these contributions are completely hydrodynamical in origin, are due to the finite size of the particles (proportional to $a^{3}$ ), and have no counterpart in molecular liquids. This last term can be expressed completely in terms of the perturbation of the structure from equilibrium by writing

$$
P_{N}=P_{N}^{\mathrm{eq}}\left(1+f_{N}\right) .
$$

The last term in Eq. (42) then becomes

$$
n k T\left\langle\mathbf{R}_{S U} \cdot \mathbf{R}_{F U}^{-1} \cdot \nabla f_{N}\right\rangle_{\mathrm{eq}},
$$

where the average is taken over the equilibrium distribution $P_{N}^{e q}$. Equation (42) may also prove to be a useful reformulation for analytic work.

We are now in a position to understand why kinetictheory-like treatments of colloidal dispersions, which completely ignore hydrodynamics, ${ }^{7,8}$ may give reasonable qualitative results. Without hydrodynamics only the first three terms on the right-hand side of Eq. (42) are present and the form of the stress tensor is identical to that of a molecular liquid. In particular, for hard spheres only the integral $\int \mathbf{n n} g(\mathbf{r}) d S$ is needed. Hence, theories or computer simulations ${ }^{20-24}$ based on molecular models will predict contributions to the stress of these same forms. While it would be highly surprising if these "molecular" theories gave quantitative predictions, they may well give reasonable qualitative dependences. At small departures from equilibrium, a simple scaling analysis shows that $\mathbf{n n} g(\mathbf{r})$ is proportional to $\langle\mathbf{E}\rangle$, proportional to $g_{\mathrm{eq}}(2)$ and inversely proportional to $D_{0}^{s}$, the short-time self-diffusivity. ${ }^{25}$ Thus, the Brownian stress contribution $\int \mathbf{n n} g(\mathbf{r}) d S$ will scale correctly with $\phi$ and Pe. It should also be expected to scale properly to higher than first order in departures from equilibrium. ${ }^{26}$

In a similar way, nonequilibrium molecular dynamics simulations or Brownian dynamics simulations (Brownian dynamics corresponds to neglecting all hydrodynamic in- 
teractions in the above formulation of particle dynamics; specifically, setting $\mathbf{R}_{S U}=\mathbf{R}_{F E}=\mathbf{R}_{S E}=0$ and $\mathbf{R}_{F U}$ $=6 \pi \eta a \mathbf{I}$ ), will also produce results for the first three terms of Eq. (42) and may be expected, therefore, to reproduce qualitative behavior at small Péclet numbers. It should be noted that not only are there additional contributions to the stress from the hydrodynamics, but the equations of motion for the particles also involve hydrodynamics and therefore the structure of the suspension may well be different if hydrodynamics are ignored. For quantitative results, clearly the hydrodynamics are important and they are essential as $\mathrm{Pe} \rightarrow \infty$ for the motion is completely dominated by hydrodynamics in this limit. It would also be interesting to see how the kinetic-theory-like descriptions fare for the high-frequency dynamic viscosity for hard spheres, which is predicted here to be purely hydrodynamic in origin.

\section{CONCLUSIONS}

We have shown in this paper that the osmotic pressure of a colloidal dispersion can be derived from a consideration of the mechanical equations of motion of the macroparticles. The mechanical equations are completely specified, in the case of hard spheres, in terms of hydrodynamic interactions, as is the expression for the bulk or macroscopic stress. This mechanical, hydrodynamical, definition of the osmotic pressure was shown to be identical to the normal thermodynamic one. This analysis places studies of supremolecular fluids on the same mechanical foundation that underlies the statistical mechanics of simple liquids. No separate appeal to thermodynamic arguments is needed as had been the case in the past. Rather, the statistical mechanics of the macroparticle fluid can be derived from the fundamental equations of motion.

With this new perspective we have been able to relate by Eq. (42) the hydrodynamic determination of the macroscopic stress, through Eqs. (36)-(39c) and implemented by Stokesian dynamics, for example, to what one has in a molecular fluid. While it has often been suspected that there should be a close analogy between molecular and macroparticle fluids, exactly what this connection is has been lacking up to now. Equation (42) shows quite clearly the connection, both its strengths and weaknesses. It also explains why kinetic-theory studies or nonequilibrium molecular dynamics or Brownian dynamics simulations may give qualitatively correct dependences for rheological response. Equation (42) may also prove to be a fruitful starting point for developing a proper theoretical description of colloidal dispersions. In particular, in our Stokesian dynamics simulations of colloidal hard spheres, we have noted that the purely hydrodynamic contribution to the stress $\left[\mathbf{S}^{H}=-\left\langle\mathbf{R}_{S U} \cdot \mathbf{R}_{F U}^{-1} \cdot \mathbf{R}_{F E}-\mathbf{R}_{S E}\right\rangle:\langle\mathbf{E}\rangle\right.$ in Eq. (38)] varies little from its value at equilibrium (which is the high-frequency dynamic viscosity) up to Péclet numbers in excess of 10. Thus, one could accept this viscosity as known and use it and the equilibrium structure as the starting point to develop a perturbation theory for sheared colloids similar to the successful perturbation theories of hard-sphere liquids. Indeed, I have carried out such a perturbation analysis and have obtained very good predictions over all volume fractions for both the magnitude and frequency dependence of the stress for Brownian hard-sphere suspensions. ${ }^{25,26}$

\section{ACKNOWLEDGMENTS}

This work was supported in part by Grant No. CTS9020646 from the National Science Foundation.

${ }^{1}$ A. Einstein, Brownian Motion (Dover, New York, 1956).

${ }^{2}$ H. T. Hill, Introduction to Statistical Mechanics (Addison-Wesley, Reading, MA, 1960).

${ }^{3}$ W. R. Russel, D. A. Saville, and W. R. Schowalter, Colloidal Dispersions (Cambridge University Press, Lóndon, 1989).

${ }^{4}$ A. Vrij, J. W. Jansen, J. K. G. Dhont, C. Pathmamanoharan, M. M. Kops-Werkoven, and H. M. Fijnaut, Faraday Discuss. Chem. Soc. 76, 19 (1983).

${ }^{5}$ C. G. de Kruif, J. W. Jansen, and A. Vrij, in Physics of Complex and Supramolecular Fluids, edited by S. A. Safran and N. Clark (Wiley, New York, 1987).

${ }^{6}$ D. A. McQuarrie, Statistical Mechanics (Harper \& Row, New York, 1976).

${ }^{7}$ E. D. G. Cohen and I. M. de Schepper, AIP Conf. Proc. 256, 359 (1992); J. Stat. Phys. 63, 241 (1991); Phys. Lett. A 150, 308 (1990).

${ }^{8}$ H. Jorquera and J. S. Dahler, J. Chem. Phys. 96, 6917 (1992).

${ }^{9}$ J. F. Brady and G. Bossis, Annu. Rev. Fluid Mech. 20, 111 (1988).

${ }^{10}$ G. Bossis and J. F. Brady, J. Chem. Phys. 91, 1866 (1989).

${ }^{11}$ L. J. Durlofsky, J. F. Brady, and G. Bossis, J. Fluid Mech. 180, 21 (1987).

${ }^{12}$ S. Kim and S. J. Karilla, Microhydradynamics (Butterworths, London, 1991).

${ }^{13}$ G. K. Batchelor, J. Fluid Mech. 83, 97 (1977).

${ }^{14}$ D. J. Jeffrey, J. F. Morris, and J. F. Brady, Phys. Fluids A (submitted).

${ }^{15}$ D. J. Jeffrey, Phys. Fluids A 4, 16 (1992).

${ }^{16}$ J. F. Brady and T. Phung, AIP Conf. Proc. 256, 391 (1992).

${ }^{17}$ R. T. Bonnecaze and J. F. Brady, J. Chem. Phys. 96, 2183 (1992); J. Rheol. 36, 73 (1992).

${ }^{18}$ G. K. Batchelor, J. Fluid Mech. 41, 545 (1970).

${ }^{19}$ R. J. Phillips, J. F. Brady, and G. Bossis, Phys. Fluids 31, 3462 (1988).

${ }^{20}$ H. A. Barnes, M. F. Edwards, and L. V. Woodcock, Chem. Eng. Sci. 42, 591 (1987).

${ }^{21}$ A. J. Hopkins and L. V. Woodcock, J. Chem. Soc. Faraday Trans. 86, 2121 (1990).

${ }^{22}$ D. M. Heyes, Phys. Lett. 132, 399 (1988).

${ }^{23}$ D. J. Evans and G. P. Morris, Statistical Mechanics of Nonequilibrium Liquids (Academic, New York, 1990).

${ }^{24}$ M. P. Allen and D. J. Tildesley, Computer Simulation of Liquids (Oxford University Press, Oxford, 1987).

${ }^{25}$ J. F. Brady, J. Chem. Phys. (submitted).

${ }^{26}$ J. F. Brady (unpublished). 